\title{
Análisis de sistemas mecánicos utilizando sistemas CAE, para estudiantes de ingeniería mecatrónica
}

\section{Analysis of mechanical systems using CAE systems, for students of mechatronic engineering}

\author{
HERNÁNDEZ-ZEMPOALTECATL，Rodrigo †*，CANO-CORONA，Ariana，PÉREZ-SERRANO, \\ Froylán y MÉNDEZ-ZAPATA, Elías
}

Universidad Politécnica de Tlaxcala, Programa Académico de Ingeniería Mecatrónica

ID $1^{\text {er }}$ Autor: Rodrigo, Hernández-Zempoaltecatl / ORC ID: 0000-0002-0677-474X

ID $1^{\text {er }}$ Coautor: Ariana, Cano-Corona / ORC ID: 0000-0003-3456-2303, CVU CONACYT ID: X_acano100364

ID $2^{\text {do }}$ Coautor: Froylán, Pérez-Serrano / ORC ID: 0000-0001-7795-7386, CVU CONACYT ID: 377744

ID $3^{\text {er }}$ Coautor: Elías, Méndez-Zapata / ORC ID: 0000-0001-5288-4432

DOI: $10.35429 /$ JOIE.2020.14.4.14.20

Recibido Abril 20, 2020; Aceptado Junio 30, 2020

\section{Resumen}

Con este trabajo se muestra que los fundamentos, de sistemas de ingeniería asistida por computadora CAE pueden ser utilizados como herramienta efectiva para la optimización del análisis de sistemas mecánicos y/o procesos de manufactura en las asignaturas de ingeniería mecatrónica. La simulación de las condiciones de operación, así como el análisis las diferentes condiciones de trabajo como el análisis de esfuerzos, los análisis térmicos, la optimización de procesos y la optimización de producto, permite que la herramienta CAE se pueda utilizar en una gran variedad de procesos desde diseños previos hasta digitalizaciones de procesos de ingeniería inversa. Durante el desarrollo de competencias tecnológicas como ingenieros mecatrónicos, las herramientas CAE son fundamentales para que los estudiantes puedan simular procesos y condiciones de operación que integran máquinas o celdas de manufactura. Ante la gran variedad de herramientas, las técnicas CAE se pueden desarrollar por diferentes métodos, sin embargo en este caso se utilizará el análisis por elementos finitos (FEA), como técnica de resolución numérica que considera que cualquier componente puede ser particularizado, en un conjunto de pequeños elementos con geometría simple y comportamiento físico conocido, generalmente este método se aplica a diferentes campos de ingeniería.

\begin{abstract}
This work shows that the fundamentals of CAE computeraided engineering systems can be used as an effective tool for optimizing the analysis of mechanical systems and / or manufacturing processes in mechatronic engineering subjects. The simulation of operating conditions, as well as the analysis of different working conditions such as stress analysis, thermal analysis, process optimization and product optimization, allows the CAE tool to be used in a wide variety of processes from previous designs to digitization of reverse engineering processes. During the development of technological skills as mechatronic engineers, CAE tools are essential for students to simulate processes and operating conditions that integrate manufacturing machines or cells. Given the wide variety of tools, CAE techniques can be developed by different methods, however in this case, finite element analysis (FEA) will be used, as a numerical resolution technique that considers that any component can be particularized, in a set of small elements with simple geometry and known physical behavior, this method is generally applied to different engineering fields.
\end{abstract} mecatrónica. Revista de Ingeniería Innovativa. 2020. 4-14:14-20.

*Correspondencia al Autor (Correo Electrónico: rodrigo.hernandez@uptlax.edu.mx)

$\dagger$ Investigador contribuyendo como primer autor. 


\section{Introducción}

Las herramientas de ingeniería asistida por computadora (CAE) consisten en el uso de software para simular los rendimientos de elementos que componen un sistema y su principal objetivo es mejorar el diseño de los productos, así como apoyar en la solución de problemas de ingeniería para diferentes sectores productivos. El uso de estas herramientas en la formación de ingenieros mecatrónicos permite que se pueda realizar simulación, validación y la optimización de productos, procesos y herramientas de fabricación.

El uso de herramientas CAE (ver Tabla 1) fortalece el desarrollo de competencias profesionales de los futuros ingenieros mecatrónicos, a través del análisis de diseños previamente realizados con softwares CAD o digitalizaciones mediante procesos de ingeniería inversa. El fortalecimiento de competencias en los estudiantes que cursan la carrera de ingeniería mecatrónica se lleva a cabo con el uso de herramientas CAE, ya que se puede extraer información para optimizar el desarrollo y los costos de fabricación de diversos elementos, reduciendo las pruebas con prototipos para la obtención del producto final.

\begin{tabular}{|c|c|c|}
\hline \multicolumn{2}{|r|}{ Software } & Características \\
\hline 1 & $\begin{array}{l}\text { Abaqus } \\
\text { Simulia }\end{array}$ & $\begin{array}{l}\text { Programa CAE de cálculo por } \\
\text { elementos finitos de propósito } \\
\text { general. }\end{array}$ \\
\hline 2 & Algor & $\begin{array}{l}\text { Solución para aplicaciones de } \\
\text { ingeniería orientada a análisis } \\
\text { computacional multifísica. }\end{array}$ \\
\hline 3 & Ansys & $\begin{array}{l}\text { Programa CAE para diseño, } \\
\text { análisis y simulación de partes } \\
\text { por elementos finitos FEA, a } \\
\text { través del malleo, ejecución } \\
\text { y post proceso, ejecuta } \\
\text { el análisis de piezas sometidas a } \\
\text { fenómenos físicos y diseño } \\
\text { mecánico, resuelve problemas } \\
\text { físicos sometidos a esfuerzos } \\
\text { térmicos, fluidos, vibración y } \\
\text { aplicaciones específicas. }\end{array}$ \\
\hline 4 & $\begin{array}{l}\text { Autodesk } \\
\text { Simulation }\end{array}$ & $\begin{array}{l}\text { Programa que valida y optimiza } \\
\text { sus diseños, simula el diseño } \\
\text { a esfuerzos virtuales para } \\
\text { predecir su comportamiento en } \\
\text { el mundo real. }\end{array}$ \\
\hline 5 & $\begin{array}{l}\text { Caedium } \\
\text { Symlab }\end{array}$ & $\begin{array}{l}\text { Solución de análisis para } \\
\text { resolver problemas de ingeniería } \\
\text { a través de la optimización de } \\
\text { modelos 3D. }\end{array}$ \\
\hline
\end{tabular}

\begin{tabular}{|c|l|l|}
\hline 6 & $\begin{array}{l}\text { Comsol } \\
\text { Multiphysics }\end{array}$ & $\begin{array}{l}\text { Software CAE para modelado, } \\
\text { análisis y simulación de } \\
\text { fenómenos físicos 3D en } \\
\text { ingeniería. }\end{array}$ \\
\hline 7 & Fe pipe & $\begin{array}{l}\text { Software para análisis por el } \\
\text { método de elementos finitos } \\
\text { pero usado exclusivamente para } \\
\text { tubería y recipientes a presión. }\end{array}$ \\
\hline 8 & Femap & $\begin{array}{l}\text { Programa de simulación y } \\
\text { análisis FEA, con pre y post } \\
\text { proceso para estimar el } \\
\text { desempeño en el desarrollo y } \\
\text { diseño de productos. }\end{array}$ \\
\hline 9 & LS-Dyna & $\begin{array}{l}\text { Programa que resuelve análisis } \\
\text { dinámico CAE de modelos 3D } \\
\text { por el método de elementos } \\
\text { finitos, para problemas reales, } \\
\text { modelos complejos no lineales y } \\
\text { cuerpos rígidos. }\end{array}$ \\
\hline 10 & $\begin{array}{l}\text { Ofrece una solución } \\
\text { completa para problemas de } \\
\text { ingeniería usando simulación y } \\
\text { Software }\end{array}$ \\
\hline
\end{tabular}

Tabla 1 Características de los principales softwares para análisis y simulación en ingeniería

Fuente: Elaboración propia

En la tabla anterior, se muestra un comparativo de las principales características de los softwares basados en herramientas CAE, sin embargo, la mayoría de ellos requieren de licencias a un costo elevado que las instituciones de educación superior no pueden solventar, de esta forma con el uso del software ANSYS se promueven metodologías de simulación que fomentan un aprendizaje significativo en los futuros profesionales.

Es importante destacar que el uso de este programa no solo se limita al cálculo de algoritmos, ya que también se obtienen resultados numéricos que permiten un análisis más integral para la toma de decisiones, en el área de Ingeniería Mecatrónica como en la simulación de flujo de fluidos, análisis de esfuerzos estructurales, transferencia de calor y determinación de secuencias mecánicas.

Con el apoyo de esta herramienta CAE en las asignaturas de ingeniería mecatrónica se pueden valorar entre otras cosas las características y propiedades de los materiales, la definición de material y optimización geométrica del modelo, así como la viabilidad y cantidad de los materiales, todo esto influirá en los costos finales de fabricación. 
Con respecto al algoritmo de los sistemas CAE, este utiliza nodos individuales en el total de la red, y cada nodo puede interactuar con otro, jugando un papel importante en el método de elementos finitos ya que utiliza la geometría del modelo existente para construir una red nodal a lo largo de él, como se observa en la figura 1.

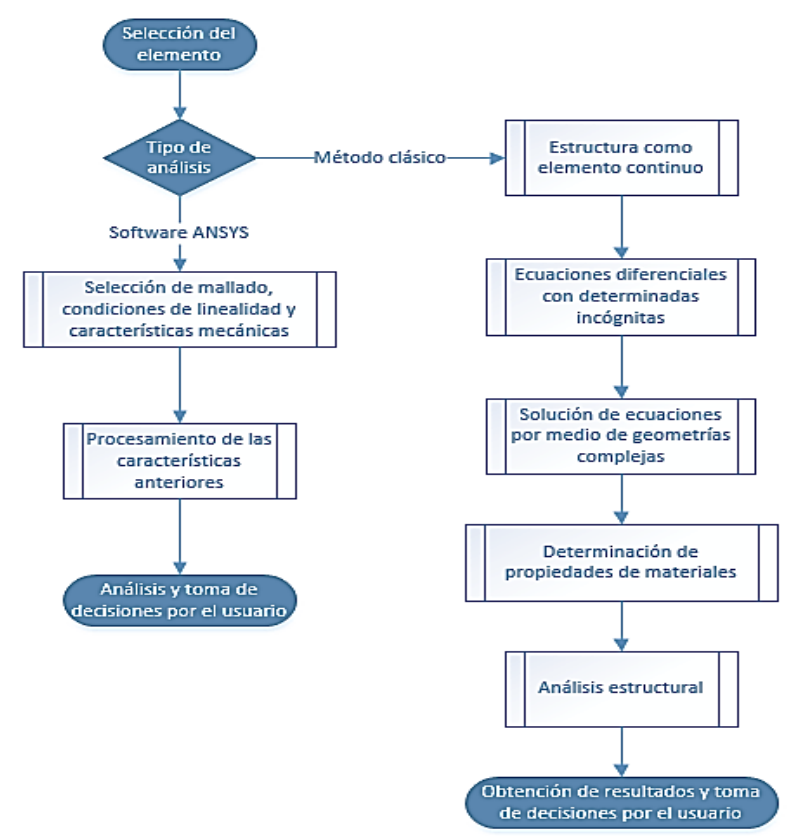

Figura 1 Metodología de análisis teórica y por software Fuente: Cano, (2016)

Entonces, la determinación del desempeño de un elemento se basa en la introducción de parámetros que la pieza experimentará en el mundo real. Los parámetros más utilizados en ingeniería mecánica para simulaciones CAE son;

\footnotetext{
- $\quad$ Temperatura.

- Presión.

- Interacciones de los componentes.

- Fuerzas aplicadas.
}

La mayoría de los parámetros que se utilizan para la simulación están basados en el ambiente y en las interacciones que el modelo debería experimentar una vez que empiece a operar y generalmente son introducidos en el programa CAE como una condición para observar si la pieza examinada podría manejar, teóricamente, las limitaciones del diseño.
Los sistemas CAE como herramienta educativa en las instituciones de educación superior se proyectan para usarlas como referencia sobre el desarrollo de procesos reales que beneficien el sector empleador. Se debe considerar que el avance exponencial de la tecnología debe servir para mejorar los procesos industriales que actualmente exigen que las simulaciones, basadas en métodos numéricos solucionen problemas con alto grado de dificultad.

\section{Aplicaciones de los sistemas CAE}

Las principales áreas de desarrollo que pueden potencializar los estudiantes a través del uso de herramientas CAE son:

- Análisis de esfuerzos y dinámica de componentes y ensambles con el empleo de MEF.

Análisis térmico y de fluidos gracias al uso de CFD.

- $\quad$ Sistema multicuerpo (MBD) y cinemática.

- Herramientas de análisis para simulación de procesos y para procesos de fabricación.

Optimización del proceso de documentación.

- $\quad$ Optimización del desarrollo del producto.

- Verificación inteligente de las inconformidades.

En general, existen tres fases en cualquier tarea de ingeniería asistida por computadora:

- Pre-proceso, que consiste en definir el modelo, así como los factores ambientales que se le aplicarán al elemento.

Análisis, que permite resolver el problema planteado.

Post procesamiento de resultados, que consiste en la toma de decisiones a partir del análisis y los resultados obtenidos. 
A continuación, mostramos algunos ejemplos de análisis utilizando como herramienta los sistemas $\mathrm{CAE}$ en las asignaturas de ingeniería mecatrónica, y como se ha mencionado anteriormente, estos análisis se pueden aplicar a un gran número de sistemas o elementos.

En el primero de ellos se muestra el análisis de la deformación total de una estructura elaborada de una viga I W356 x 171 utilizando el software Ansys.

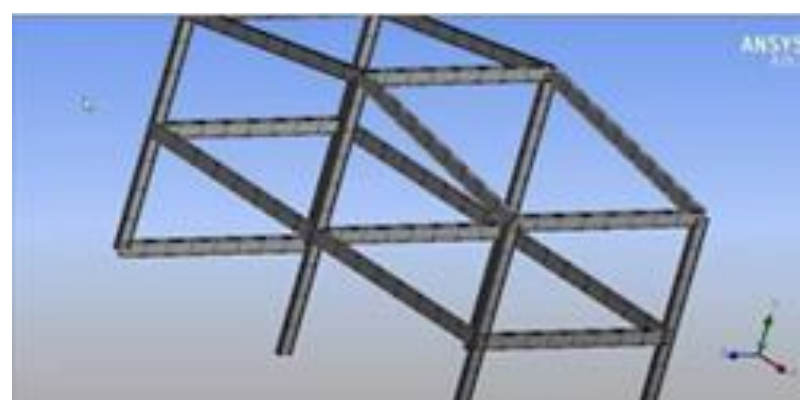

Figura 2 Estructura metálica

Fuente: Elaboración propia con software Ansys

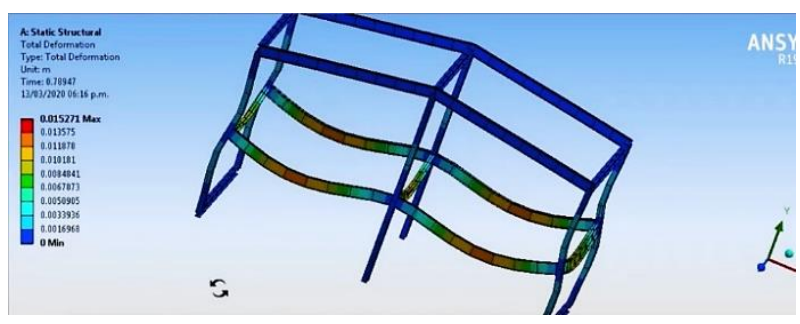

Figura 3 Análisis de la deformación total

Fuente: Análisis propio con Ansys

Como se puede observar en las figuras 2 y 3 , el software permite tomar decisiones a partir de la simulación de esfuerzos sobre las estructuras, además de personalizar las condiciones sobre las cuales trabajarían, por lo tanto, se tiene una ventaja muy grande con los estudiantes al poder analizar múltiples escenarios y decidir las mejores opciones de trabajo de los sistemas.

Para el segundo ejemplo, utilizamos una tolva para la separación de polvos, donde se puede observar el flujo del aire que entra tangencialmente a la tolva y por gravedad cae el polvo.

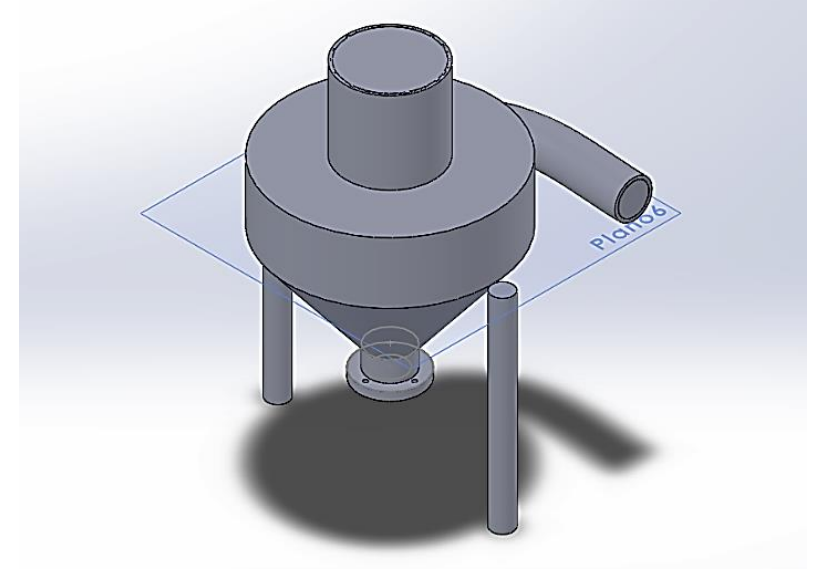

Figura 4 Tolva para separación de polvos

Fuente: Elaboración propia con Solid Works

Como se observa en la siguiente figura (5), con el uso del software y las herramientas implícitas en el mismo, se pueden simular las corrientes del aire y los efectos que estas provocarían bajo ciertas condiciones de trabajo.

Estos modelos generados en el software, permiten que los estudiantes simplifiquen costos de producción y detallar condiciones que se podrían presentar en casos reales, por lo tanto nos ayuda al desarrollo de capacidades de diseño, desarrollo y análisis de sistemas mecánicos.

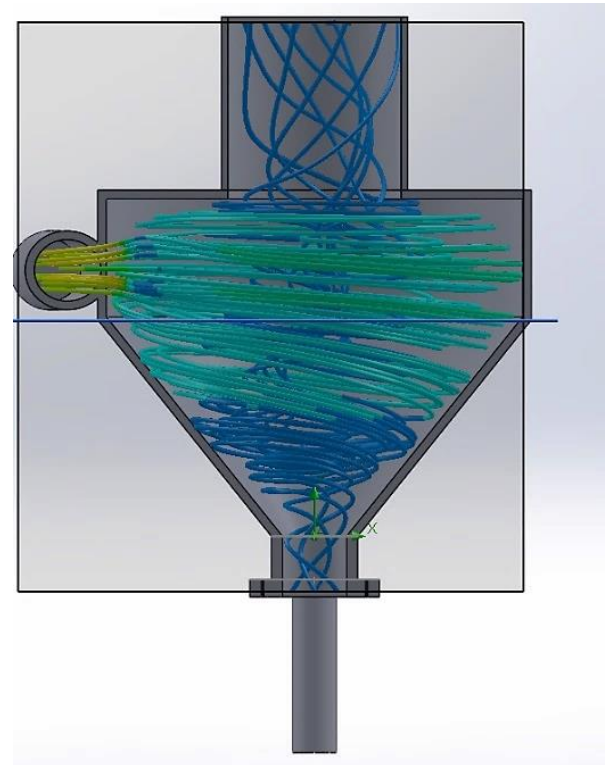

Figura 5 Vista frontal del comportamiento del flujo de aire

Fuente: Elaboración propia con SolidWorks 


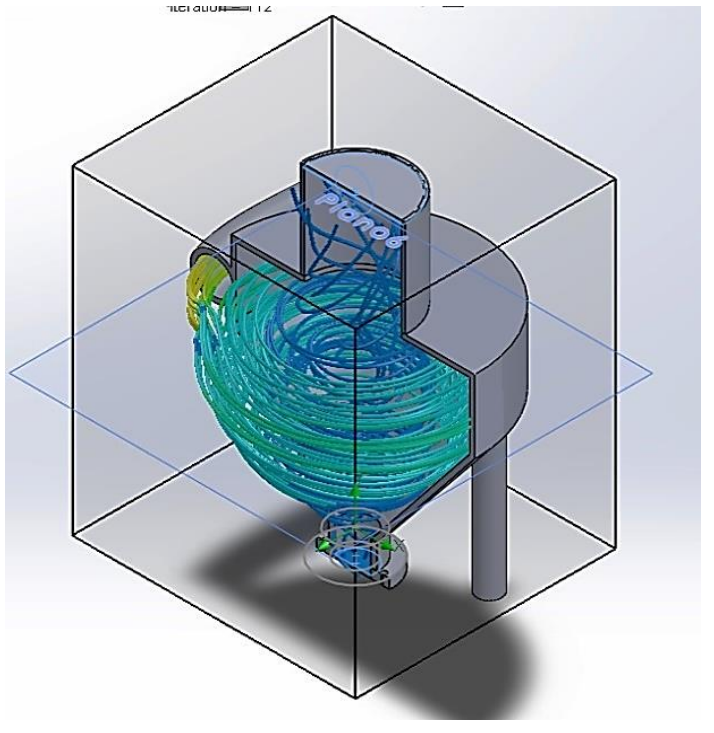

Figura 6 Vista isométrica del comportamiento del flujo de aire

Fuente: Elaboración propia con SolidWorks

\section{Desarrollo y aplicación de Sistemas CAE a un elemento mecánico}

\section{Esfuerzos Térmicos en una viga en voladizo}

Para llevar a la aplicación el uso de la herramienta CAE se propone a estudiantes de 6to cuatrimestre de ingeniería mecatrónica que analicen los esfuerzos térmicos de una viga en voladizo, ya que los voladizos se encuentran frecuentemente en diversos tipos de construcciones, especialmente en puentes de voladizo y algunos balcones.

El planteamiento del problema es el siguiente; una barra de acero $(\mathrm{E}=2.0 \mathrm{E} 10 \mathrm{~Pa}, v$ $=0.3, \alpha=1.2 \mathrm{E}-5)$ con las dimensiones que se muestran se coloca entre dos paredes. Por un lado, la barra está rígidamente fijada a la pared y por el otro, hay un espacio de $2 \mathrm{~mm}$ entre la pared y la barra. ¿Cuál es el esfuerzo en la barra después de que aumenta la temperatura $100{ }^{\circ} \mathrm{C}$ ?

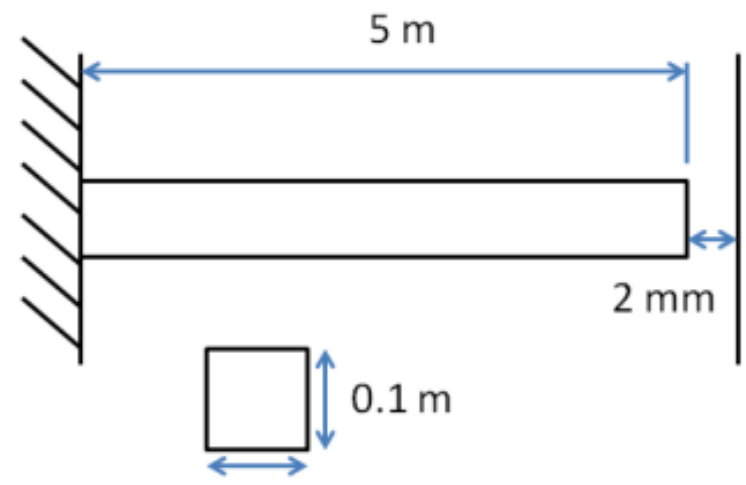

$0.1 \mathrm{~m}$

Figura 7 Descripción de la viga en voladizo

Fuente: Elaboración propia

\section{Análisis de los Esfuerzos Térmicos}

Primero, se determina si la expansión de la barra es mayor que el espacio libre entre la barra y la pared $\mathrm{x}=0.002$ metros.

$\delta_{T}=\alpha L \Delta T$

Si $\delta_{T}>x$, entonces sabemos que habrá tensión en la barra. De lo contrario, el esfuerzo en la barra $\sigma_{x x}=0$. Insertando los valores, encontramos:

$$
\delta_{T}=\left(1.2 \times 10^{-5 \circ} \mathrm{C}^{-1}\right)(5 \mathrm{~m})\left(100^{\circ} \mathrm{C}\right)=.006 \mathrm{~m}>.002
$$

Ahora que sabemos que la deformación debida al cambio de temperatura será mayor que el espacio entre la pared y la barra, sabemos que habrá una tensión en la barra.

Del problema podemos observar que, si la pared no estuviera allí, la barra se deformaría $\delta_{T}$. Sin embargo, debido a que la pared está allí, la barra no deformará la $\delta_{T}$ completa. Podemos dividir aún más el problema en esta ecuación.

$\delta_{T}=x+\delta_{\sigma}$

Donde $\delta_{T}$ es la contribución de deformación del cambio de temperatura y $\delta_{\sigma}$ es la contribución de deformación de la fuerza impartida por la pared sobre la barra. Por lo tanto:

$\alpha L \Delta T=x+\frac{P L}{E A}$

Sustituyendo $\sigma_{x x}=\frac{P}{A}$, obtenemos una ecuación solucionable

$\alpha L \Delta T=x+\frac{\sigma_{x x} L}{E}$

Resolviendo para $\sigma$, encontramos que

$\sigma_{x x}=\alpha E \Delta T-\frac{x E}{L}$

Después de sustituir, encontramos

$$
\begin{aligned}
& \sigma_{x x}=\left(1.2 \times 10^{-5 \circ} \mathrm{C}^{-1}\right)\left(2 \times 10^{11} \mathrm{~Pa}\right)\left(100^{\circ} \mathrm{C}\right)-\frac{.002 \mathrm{~m}\left(2 \times 10^{11} \mathrm{~Pa}\right)}{5 \mathrm{~m}} \\
& \sigma_{x x}=160 \mathrm{MPa}
\end{aligned}
$$




\section{Resultados aplicando Ansys Workbench como herramienta de sistemas CAE}

Una vez que realizamos los cálculos previos de forma tradicional, utilizaremos la simulación en ANSYS Workbench para mostrar la rapidez y ventajas de las herramientas CAE en la formación de los futuros ingenieros mecatrónicos.

La temperatura obtenida fue de 122 grados centígrados, que es lo que se esperaba con los cálculos tradicionales y lo podemos observar en la figura 8.

De esta manera, se comprueba que el uso del software como herramienta pedagógica, nos provee de elementos para el análisis de resistencia de piezas y estructuras que integran sistemas mecánicos más complejos.

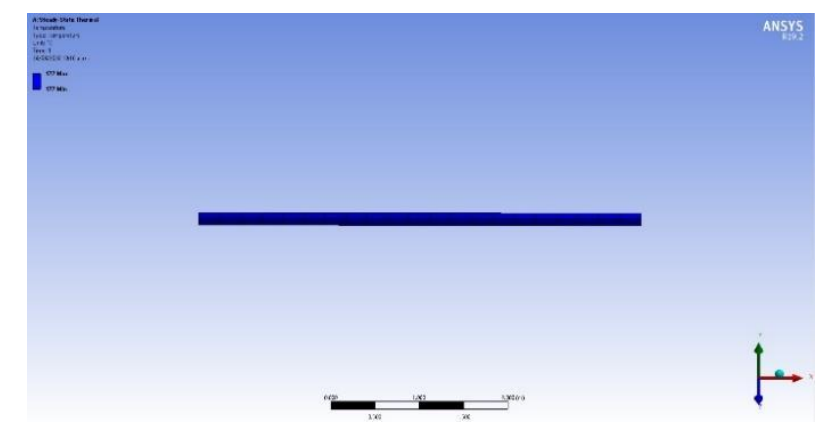

Figura 8 Temperatura obtenida de la viga

Fuente: Análisis propio con Ansys

El valor de temperatura obtenido y las cargas físicas a las que se somete a la viga, nos ayudan a obtener la deformación máxima que presenta la viga es $.002 \mathrm{~m}$ (mostrada en la figura 9), lo cual también es coincidente con la condición de frontera.

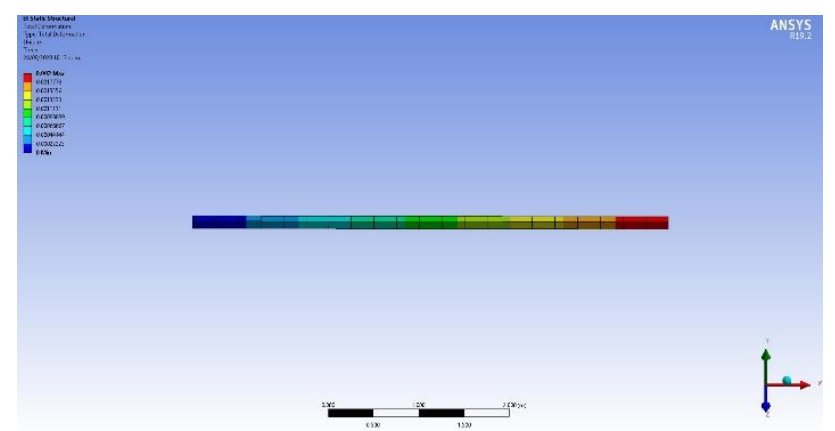

Figura 9 Deformación total de la viga Fuente: Análisis propio con Ansys
Finalmente, en la figura 10, con el apoyo del software observamos que la respuesta del esfuerzo es constante en todo momento y por lo tanto los alumnos podrían predecir el rendimiento de esta estructura en servicio.

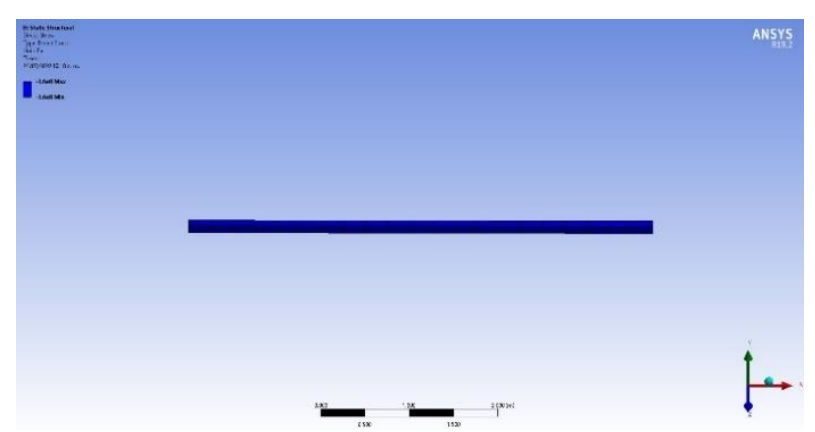

Figura 10 Resultado del esfuerzo Normal

Fuente: Análisis propio con Ansys

Para la validación y comparación de resultados, es importante verificar la simulación. Sin verificación, no se tendrían evidencias de que la simulación es confiable, por lo tanto, en la tabla 2 comparamos el resultado obtenido con la herramienta CAE y el esfuerzo obtenido con el análisis teórico que establece la mecánica de materiales.

\begin{tabular}{|r|r|r|}
\hline & Análisis teórico & Simulación \\
\hline Esfuerzo & $-1.6 \mathrm{E} 8 \mathrm{~Pa}$ & $-1.6 \mathrm{E} 8 \mathrm{~Pa}$ \\
\hline
\end{tabular}

Tabla 2 Resultados con análisis teórico y simulación con Ansys Workbench

Fuente: Elaboración propia

Observamos que el análisis sobre la intensidad de las fuerzas, muestra que el esfuerzo en la viga es coincidente de forma perfecta con la simulación realizada con el software, por lo tanto se tomaría la decisión de que no es necesario de refinar más la malla.

\section{Agradecimientos}

A la Universidad Politécnica de Tlaxcala, por las facilidades otorgadas para la realización del trabajo.

\section{Conclusiones}

Con este trabajo se observa y muestra que las tecnologías de los sistemas de ingeniería asistida por computadora CAE como herramienta educativa en los estudiantes de ingeniería mecatrónica presenta varias ventajas. 
El uso de esta herramienta en diversos sistemas permite que de manera gráfica los alumnos tengan una referencia de las condiciones de operación y en su caso de falla de los elementos o sistemas para así poder optimizar los sistemas o procesos antes de fabricarlo.

Estas herramientas tecnológicas se encuentran ya en una fase de madurez en varias asignaturas de la carrera de ingeniería mecatrónica, ya que su utilidad es indiscutible y han abierto muchas posibilidades para el rediseño y fabricación que serían impensables sin estas herramientas.

La aplicación comercial de los diferentes softwares CAE, como Ansys ha sido de ayuda invaluable, porque permite a ingenieros $y$ estudiantes la misma oportunidad de desarrollar proyectos de gran complejidad.

Por lo tanto, el uso de estas herramientas ha facilitado mucho la tarea de los estudiantes de Ingeniería Mecatrónica para realizar análisis y diseño de sistemas mecánicos, complementando los procesos de enseñanza en las aulas, brindando herramientas más gráficas para la interpretación de resultados y por lo tanto panoramas más amplios del comportamiento de los prototipos.

Es importante destacar que este tipo de herramientas también facilita el proceso de simulación de los ensamblajes de los proyectos interdisciplinarios que se realizan por parte de los alumnos al término de cada cuatrimestre, promoviendo el logro de las competencias propuestas para cada ciclo de formación.

Finalmente consideramos que, de acuerdo con la demanda del sector laboral de la región, es recomendable que los alumnos dominen el uso de estas herramientas de diseño para solventar las necesidades profesionales de los diferentes sectores industriales.

\section{Referencias}

Cano Corona A., Hernández Zempoaltecatl R. (2016) Análisis de esfuerzos en ensamblajes mecánicos, utilizando ANSYS. Congreso Internacional de Investigación de AcademiaJournals.com, Tlaxcala, México.
David W. Nicholson. Finite Element Analysis Thermomechanics of Solids. 2003 by CRC CRC Press LLC

Gary B. Bertoline. Technical graphics communication. Fourth edition. Copyright () 2009 by The McGraw-Hill Companies, Inc.

Hamri, Okba \& Léon, Jean-Claude \& Giannini, Franca \& Falcidieno, Bianca. (2010). Method, models and tools for CAD-CAE integration. Recent Patents on Mechanical Engineering. 3. 106-130.

Hernández Zempoaltecatl Rodrigo, Cano Corona Ariana, Mendez Zapata Elías y Pérez Serrano Froylan. Análisis bi-dimensional de una viga, por el método de aproximaciones planas, usando ANSYS. Revista de Tecnología e Innovación. 2017, México.

James D. Bethune. Engineering Design and Graphics with SolidWorks ${ }^{\circledR}$ 2019. Pearson Education.

Larry J. Segerlind. Applied Finite Element Analysis. Second Edition 1984 by Jhon Wiley \& Sons, Inc.

Shigley J.E., Mitchell Larry D. Mechanical Engineering Design. Mc Graw Hill. Cuarta edición. México, (1986).

Steven R. Schmid, Bernard J. Hamrock., Bo O. Jacobson. Fundamentals of Machine Elements. Third Edition. 